\title{
Increased expression of calpain 6 during the progression of uterine cervical neoplasia: Immunohistochemical analysis
}

\author{
SUN-JOO LEE ${ }^{1}$, BYOUNG-GIE KIM ${ }^{2}$, YOON-LA CHOI ${ }^{3}$ and JEONG-WON LEE ${ }^{2}$ \\ ${ }^{1}$ Department of Obstetrics and Gynecology, Konkuk University Hospital, Konkuk University School of Medicine, \\ Seoul 143-729; Departments of ${ }^{2}$ Obstetrics and Gynecology, ${ }^{3}$ Pathology, Samsung Medical Center, \\ Sungkyunkwan University School of Medicine, Seoul 135-710, Korea
}

Received August 28, 2007; Accepted December 11, 2007

\begin{abstract}
Calpain 6 (Capn6) is one of the calcium-dependent intracellular nonlysosomal proteases and is known for tissuespecific expression, primarily in the placenta. We investigated whether the expression of Capn6 is associated with the progression of uterine cervical neoplasia. We analyzed 81 formalin-fixed paraffin-embedded cervical tissues that included six normal cervical epithelium, 20 low-grade squamous intraepithelial lesions (LSIL), 17 high-grade squamous intraepithelial lesions (HSIL), 20 invasive squamous cell carcinomas (ISCC) without lymph node (LN) metastasis and 18 ISCCs with LN metastasis. The expression of Capn6 was determined by immunohistochemistry and was undetectable in normal cervical squamous epithelium. The expression of the protein gradually increased in accordance with the progression from normal to LSIL $(\mathrm{P}=0.000)$ and from LSIL to HSIL $(\mathrm{P}=0.003)$. Capn6 was detected in all cases of ISCC. However, there was no significant difference between HSIL and ISCC $(\mathrm{P}=0.945)$, ISCC with LN metastases and ISCC without LN metastases $(\mathrm{P}=0.862)$. The distribution of staining was diffuse. The cytoplasm and nucleus were stained evenly. These results suggest that Capn6 may play a significant role in the development of LSIL and may also function in the progression of LSIL to HSIL. However, its role in tumor development and metastases is not clear.
\end{abstract}

\section{Introduction}

Cervical cancer is the result of a multi-step process involving a sequential transition from normal epithelium to preneoplastic

Correspondence to: Dr Byoung-Gie Kim, Department of Obstetrics and Gynecology, Samsung Medical Center, Sungkyunkwan University School of Medicine, Gangnam-Gu Irwon-dong 50, Seoul 135-710, Korea

E-mail: bksong.kim@samsung.com

Key words: uterine cervical neoplasms, cervical intraepithelial neoplasia, calpain 6 protein, immunohistochemistry, squamous cell carcinoma cervical intraepithelial neoplasia (CIN) and then to invasive cervical cancer (1). Although it is well accepted that high-risk human papillomaviruses (HPVs) are associated with cervical cancer (1), HPV infection alone is insufficient for malignant transformation and specific cellular gene alterations are also required for the development of cancer. Identification of these genes has important implications for cervical cancer screening and treatment.

Calpains constitute a family of cytosolic calciumdependent cysteine proteases. Several mammalian isoforms of calpain (Capn1-13) that are widely expressed in many tissues have been described (2). The classic calpain proteases consist of an isoform-specific large subunit and an invariant small subunit (3). The large $80 \mathrm{kDa}$ subunit of Capn2 can be subdivided into four domains (D-I to D-IV) and the small $30 \mathrm{kDa}$ subunit consists of two domains, D-V and D-VI. D-IV and D-VI have been proposed to confer calcium dependency on the activity of calpain (4). The physiological function of calpains is unclear; however, it has been postulated that calpains are involved in cell migration through alteration of the architecture of cell adhesion and cytoskeletal components and through involvement in intracellular signaling pathways (5).

Capn6 possesses most of the residues conserved in calpain family members; however, the C-terminal region of Capn6 lacks homology to the calmodulin-like domain (D-IV) present in other vertebrate calpains $(6,7)$ and is referred to as Domain T (D-T). The D-T domain lacks any site conforming to the EF-hand $\left(\mathrm{Ca}^{2+}\right.$-binding) consensus sequence, suggesting that it may not bind $\mathrm{Ca}^{2+}$. Moreover, in humans the amino acids that constitute the canonical active site of the predicted Capn6 protein, Cys, His and Asn, are modified to Lys, His, and Asn, making it unlikely that Capn6 is proteolytically active (7). Capn6 was recently shown to be a microtubulestabilizing protein that is expressed in embryonic tissues and may be involved in the regulation of microtubule dynamics and cytoskeletal organization. In Capn6 transfected cells, cytokinesis was retarded and was often aborted to yield multinucleated cells (8).

Capn6 has much more tissue-specific expression in adults than the ubiquitously expressed Capn1 and Capn2. Although it is expressed in a number of embryonic tissues, it has been found primarily in the placenta in adults. In 1997, it was reported that Capn6 mRNA was expressed only in the placenta out of 50 normal tissue RNAs tested (6). In 2003, the Capn6 
gene was found to be overexpressed in leiomyomas compared with normal myometrium by gene-expression analysis and was either not expressed or expressed at very low levels in 18 other tissue types (9). Few reports exist in the literature on Capn6 expression in human cancer. Skubitz et al used microarray analysis to show that Capn6 was expressed in two of four uterine leiomyosarcoma (LMS) samples and in one of the four non-uterine LMS samples (10). We previously reported expression of Capn6 in endometrial stromal sarcomas, malignant mullerian mixed tumors and uterine LMS with no association to tumor stage and survival (11). As a continuation of our previous study, we further studied Capn6 expression in several other gynecological malignant samples, including cervical cancer. To investigate whether an altered expression of Capn6 is associated with neoplastic development and/or progression in the uterine cervix, we evaluated Capn6 expression in biopsy samples corresponding to normal cervical epithelium, CIN lesion and invasive squamous cell carcinoma (ISCC). We present data showing that expression of Capn6 increases in parallel with the progression of cervical neoplasia.

\section{Materials and methods}

Tissue samples. A total of 81 tissue specimens, including 20 low-grade squamous intraepithelial lesions (LSIL), 17 highgrade squamous intraepithelial lesions (HSIL), 20 ISCC without lymph node (LN) metastasis, 18 ISCC with LN metastasis and six normal specimens from the cervix were obtained from the Department of Pathology, Samsung Medical Center, Sungkyunkwan University School of Medicine. All tissue specimens were obtained from conization procedures or radical hysterectomies of 81 independent patients from 1994 to 2004. Two gynecological pathologists reviewed all the cases in regard to the histological type, histological grading and LN status. Three-micrometer-thick sections were cut from formalin-fixed, paraffin-embedded blocks and were applied to routine histopathology slides and immunohistochemistry slides. Representative tumor sections including adjacent non-tumor mucosa were analyzed by immunohistochemistry.

Immunohistochemical analysis. Formalin-fixed paraffinembedded tissue sections were immunostained with antiCapn6 D-T (anti-DT) polyclonal antibody (1:50 dilution, Affinity BioReagents, Deerfield, IL) using a two-step EnVision technique. Paraffin sections were deparaffinized and rehydrated. Endogenous peroxidase activity was blocked with $3 \%$ hydrogen peroxide. Microwave antigen retrieval (2 x $15 \mathrm{~min}$ ) was performed in citric acid ( $\mathrm{pH}$ 6.0). Following overnight incubation at $4^{\circ} \mathrm{C}$ with the primary rabbit polyclonal antibodies, slides were incubated with an EnVision kit (Dako, Copenhagen, Denmark) for $30 \mathrm{~min}$ at room temperature. Specimens were then stained with diaminobenzidine and counterstained with Mayer hematoxylin. Normal myometrial tissue was used as a negative control and placental tissue as a positive control (Fig. 1). The analysis was performed in a semiquantitative manner: for each specimen an immunohistochemical score from 0 to 3 was determined based on the staining intensity. Positive controls were designated as moderate staining and scored as $2^{+}$. The staining intensity of specimens was evaluated as follows: no staining as 0 , weaker than the positive control as $1^{+}$, equivalent to the positivecontrol as $2^{+}$and stronger than the positive control as $3^{+}$. Slides were scored independently by two pathologists (Y.L.C. and G.H.A.) and a consensus score was obtained for each slide.

Statistical analysis. Statistical analyses were performed using SPSS for Windows version 11.0. The Jonckheere-Terpstra test was applied to correlate the expression of Capn 6 with progression from normal cervical tissue to ISCC with LN metastasis and the least significance test using ranks was applied for multiple comparisons between the five groups. A $\mathrm{P}$-value of $<0.05$ was considered statistically significant.

\section{Results}

Clinical data. The median ages of patients with the different histological types were 44, 39, 38, 51 and 49 years for normal, LSIL, HSIL, ISCC without LN metastasis and ISCC with LN metastasis, respectively (Table I).

Immunohistochemistry. Capn6 expression was undetectable in normal cervical squamous epithelium of all control samples; however, the basal layer of a normal endocervical gland exhibited nuclear and cytoplasmic Capn6 staining (Fig. 1) and some of the fibroblasts and endothelial cells in the stroma of the cervix showed immunoreactivity against anti-DT. If the results were restricted to the squamous cell layers of the normal cervix, all control cases showed negative staining for Capn6. In contrast, Capn6 was detected in all cases of ISCC regardless of lymph node metastasis (Table II, Figs. 1 and 2). Capn6 staining was diffuse and the cytoplasm and nucleus appeared to stain similarly. For LSIL, expression of Capn6 was also observed in $100 \%(20 / 20)$ of the cases. However, none of the LSIL slides stained strongly and 18 of the LSIL cases $(90 \%)$ stained weakly $\left(1^{+}\right)$with a similar pattern to ISCC (Table II). All HSIL cases (17/17) demonstrated expression of Capn6 (Table II, Figs. 1 and 2); four cases (24\%) stained strongly and eight cases (47\%) stained weakly. When the four grades (normal, LSIL, HSIL and ISCC) were compared with respect to the intensity of staining, the expression of Capn6 gradually increased in accordance with the progression from normal to LSIL to HSIL (normal vs. LSIL, $\mathrm{P}=0.000$; LSIL vs. HSIL, $\mathrm{P}=0.003$ ) (Table II, Figs. 1 and 2). There was a statistically significant difference between LSIL and ISCC $(\mathrm{P}=0.000)$, though not between HSIL and ISCC $(\mathrm{P}=0.945)$. In addition, there was no significant difference between ISCC with LN metastases and ISCC without LN metastases $(\mathrm{P}=0.862)$.

\section{Discussion}

This is the first report describing the expression of Capn6 during the progression of human uterine cervical neoplasia. Although the physiological function of Capn6 remains unknown, the results of this study indicate that Capn6 may be involved in neoplastic progression in the uterine cervix. The current study demonstrated that Capn6 was expressed in all uterine cervical neoplasias (LSIL, HSIL and ISCC) examined, in contrast to the negative expression in all normal 
Table I. Patient characteristics.

\begin{tabular}{|c|c|c|c|c|c|}
\hline Characteristics & $\begin{array}{l}\text { Normal } \\
(n=6)\end{array}$ & $\begin{array}{c}\text { LSIL } \\
(n=20)\end{array}$ & $\begin{array}{l}\text { HSIL } \\
(n=17)\end{array}$ & $\begin{array}{l}\text { ISCC, } \\
\text { LN (-) } \\
(n=20)\end{array}$ & $\begin{array}{l}\text { ISCC, } \\
\mathrm{LN}(+) \\
(\mathrm{n}=18)\end{array}$ \\
\hline \multicolumn{6}{|l|}{ Age (yr) } \\
\hline Median & 44 & 39 & 38 & 51 & 49 \\
\hline Range & $36-53$ & $26-56$ & $28-47$ & $28-72$ & $29-71$ \\
\hline Menopause & $2 / 6(33 \%)$ & $1 / 20(5 \%)$ & 0/17 (0\%) & $11 / 20(55 \%)$ & $7 / 20(35 \%)$ \\
\hline \multicolumn{6}{|l|}{ Stage } \\
\hline Ib1 & & & & 6 & 6 \\
\hline Ib2 & & & & 5 & 6 \\
\hline IIa & & & & 9 & 6 \\
\hline
\end{tabular}

LSIL, low-grade squamous intraepithelial lesion; HSIL, high-grade squamous intraepithelial lesion; ISCC LN (-), invasive squamous cell carcinoma without lymph node metastasis; ISCC LN (+), invasive squamous cell carcinoma with lymph node metastasis.

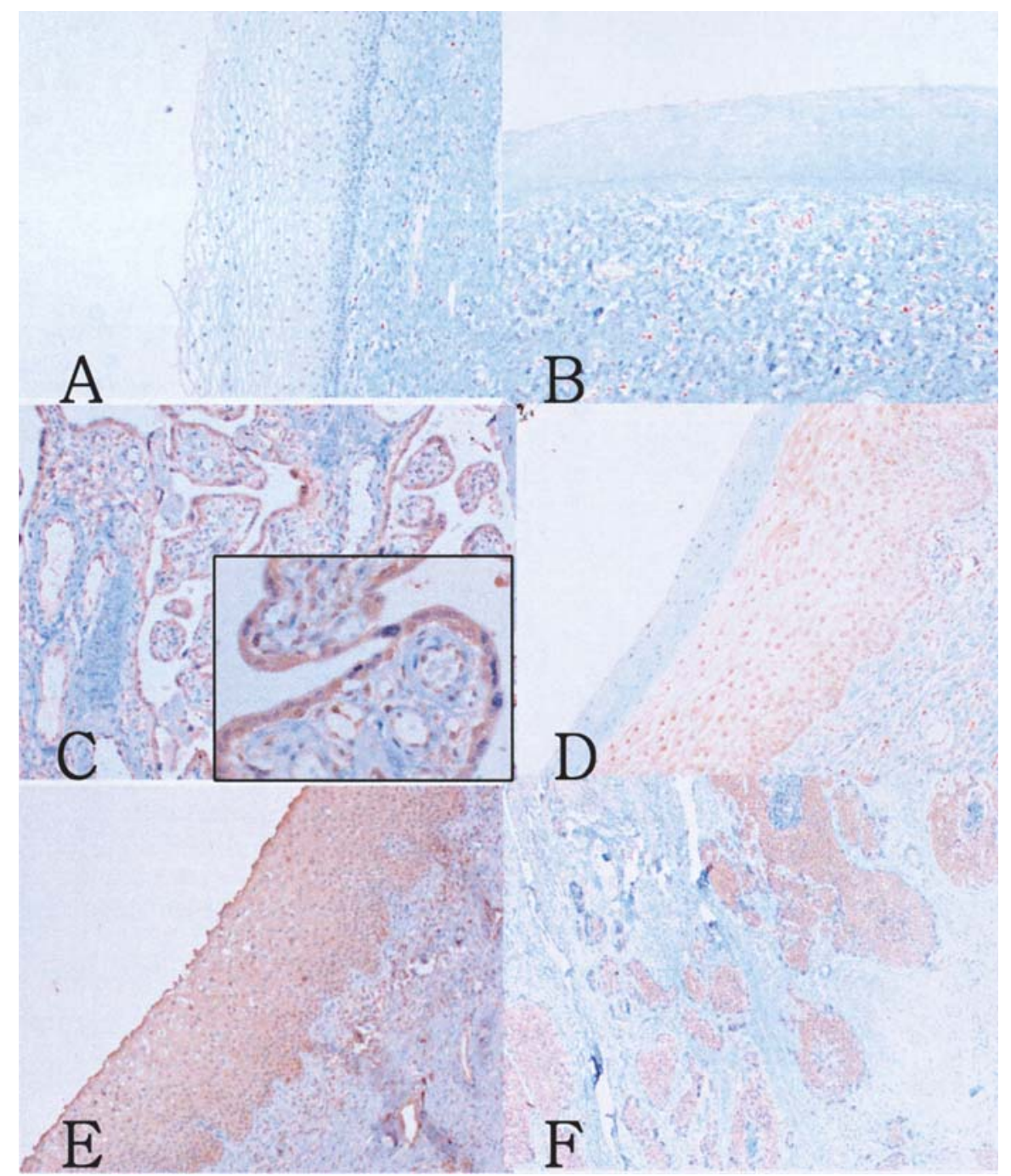

Figure 1. Immunohistochemical staining of anti-Capn6 D-T antibodies. (A,B) Normal cervical squamous epithelium showing no immunostaining. (C) Normal placenta (score 2, magnification, x100; inlet, magnification, x400), positive staining. (D) Low-grade squamous intraepithelial lesion (LSIL) showing $1^{+}$ immunostaining. (E) High-grade squamous intraepithelial lesion (HSIL) showing $2^{+}$immunostaing. (F) Invasive squamous cell carcinoma (ISCC) showing $2^{+}$ immunostaining. 
Table II. The expression of calpain 6 in normal cervical squamous epithelium, low-grade squamous intraepithelial lesion (LSIL), high-grade squamous intraepithelial lesion (HSIL), invasive squamous cell carcinoma (ISCC) without lymph node (LN) metastasis and ISCC with LN metastasis.

Expression of calpain 6

\begin{tabular}{lcccc}
\cline { 2 - 5 } Histological grade & 0 & $1^{+}$ & $2^{+}$ & $3^{+}$ \\
\hline Normal $^{\mathrm{a}}$ & $6 / 6(100 \%)$ & - & - & - \\
LSIL $^{\mathrm{b}}$ & - & $18 / 20(90 \%)$ & $2 / 20(10 \%)$ & - \\
HSIL $^{\mathrm{c}}$ & - & $8 / 17(47 \%)$ & $5 / 17(29 \%)$ & $4 / 17(24 \%)$ \\
ISCC, LN (-) & - & $9 / 20(45 \%)$ & $7 / 20(35 \%)$ & $4 / 20(20 \%)$ \\
ISCC, LN $(+)^{\mathrm{e}}$ & - & $7 / 18(39 \%)$ & $8 / 18(44 \%)$ & $3 / 18(17 \%)$ \\
\hline
\end{tabular}

${ }^{a-b}(P=0.000) ;{ }^{b-c}(P=0.003) ;{ }^{c-d, e}(P=0.945) ;{ }^{b-d, e}(P=0.000) ;{ }^{d-e}(P=0.862)$.

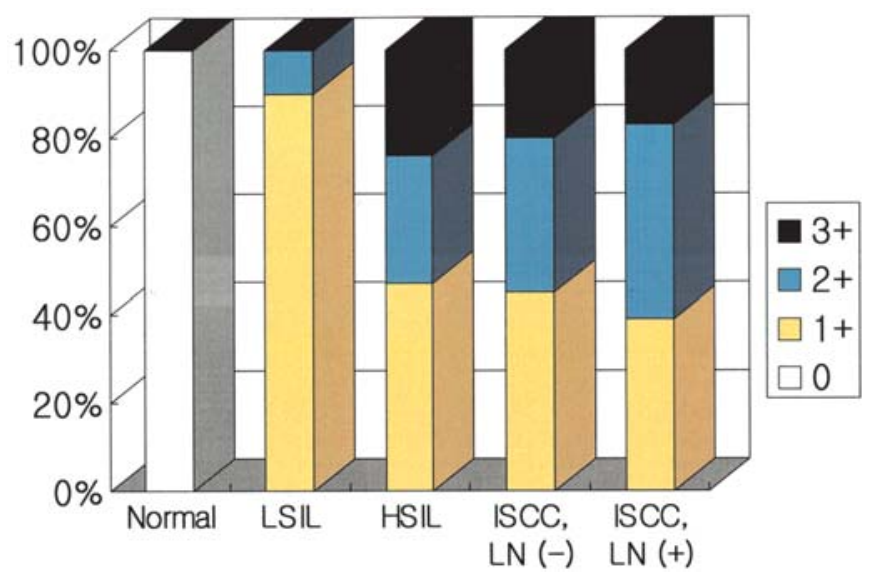

Figure 2. Percentage of scoring of immunohistochemical staining of calpain 6 in normal cervical squamous epithelium, low-grade squamous intraepithelial lesion (LSIL), high-grade squamous intraepithelial lesion (HSIL), invasive squamous cell carcinoma (ISCC) without lymph node (LN) metastasis and ISCC with $\mathrm{LN}$ metastasis $(\mathrm{P}<0.01)$.

cervical squamous cell epithelium samples (Table II, Figs. 1 and 2). The expression of Capn6 was observed from the early stages in the progression of cervical neoplasia and increased significantly with stage progression from LSIL to HSIL and ISCC (Table II, Figs. 1 and 2). Protein expression was evident in all cases of HSIL and ISCC and there was no significant difference in expression between these two stages. These results suggest that expression of Capn6 begins early during disease progression and is associated with disease progression of CIN rather than the malignant transformation of the cervical squamous cells. However, it should be noted that the cases of normal epithelium included in our study did not show the transformation zone and further studies with normal tissues containing a transformation zone are required.

It is well known that cases of LSIL are heterogeneous with respect to natural history and viral presence. While the majority of LSIL regress during long-term follow-up, between 8 and $25 \%$ progress to HSIL and occasionally to invasive cancer $(12,13)$. Management of women with LSIL is currently limited by the inability to predict which patients are at risk of progression. The ability to predict the risk of progression using markers that identify genetic variants associated with transformation or HPV genomes and proteins associated with transformation would greatly facilitate prevention and early intervention (14). In addition to HPV variants, candidate markers currently being studied include HPV, E6 and E7 messenger RNA, FHIT, p16, loss of heterozygosity at specific chromosomal loci and DNA ploidy (15-19). Although there is an expectation that one or more markers associated with a high-risk of progression will be identified, there is currently no such marker that can be clinically applied.

In the current study, we found that $90 \%$ of LSIL cases stained weakly with anti-DT antibody and none stained strongly. In contrast, $24 \%$ of the HSIL cases and $18 \%$ of the invasive cervical cancers stained strongly with the anti-DT antibody. Thus, it can be hypothesized that increased expression of Capn6 is one genetic alteration required for the progression from LSIL to HSIL and that the expression of Capn6 may be used as a biomarker to predict which patients with LSIL are at highest risk of disease progression. Our data also indicate that Capn6 expression is not associated with age or hormone status (Table I), although further evaluations with larger sample sizes are necessary. One clinical application would be to monitor Capn6 expression during a follow-up of patients with LSIL who are not receiving therapy to predict the risk of disease progression. Therefore, a prospective observational study to elucidate the role of Capn6 in the progression of LSIL patients is warranted. An understanding of the role of Capn6 in uterine cervical neoplasia will likely provide insight into cervical carcinogenesis and clues for identifying strategies for a better diagnosis, treatment and prevention of cervical cancer. Capn6 has recently been shown to be a microtubulestabilizing protein expressed in embryonic tissues and may be involved in the regulation of microtubule dynamics and cytoskeletal organization (8). Cytokinesis was retarded in Capn6 transfected cells and was often aborted producing multinucleated cells. The altered regulation of microtubule dynamics and cytoskeletal organization by Capn6 may be associated with cervical neoplasia development and/or progression.

The classic calpains are abundant in the cytoplasm of the cell with a slight predominance around the nucleus (20). These 
proteases can cleave many intracellular signaling and structural proteins and active calpains have been found predominately in the plasma membrane, indicating that membrane localization may regulate calpain activity (5). A previous study reported that Capn6 was mainly distributed in the perinuclear region and that the localization largely overlapped with thick microtubule bundles (8). Notably, our study revealed an even distribution of Capn6 between the cytoplasm and nucleus within the tumor cells. Further experiments using Western blot or RT-PCR analysis on cytoplasmic and nuclear fractions are required to confirm the nuclear expression of Capn6. At the present time, we are unable to exclude the possibility of non-specific binding of antibodies to the nucleus.

In conclusion, this study demonstrated that the expression of Capn6 increased during the progression of cervical neoplasia from the early stages of LSIL to ISCC, indicating that Capn6 might play an important role in the progression of uterine cervical neoplasia. Further studies are necessary in order to elucidate the role of Capn6 in tumor progression.

\section{Acknowledgements}

This study was supported by Konkuk University in 2008.

\section{References}

1. Richart RM: A modified terminology for cervical intraepithelial neoplasia. Obstet Gynecol 75: 131-133, 1990.

2. Sorimachi H, Ishiura S and Suzuki K: Structure and physiological function of calpains. Biochem J 328: 721-732, 1997.

3. Dear TN and Boehm T: Identification and characterization of two novel calpain large subunit genes. Gene 274: 245-252, 2001.

4. Carragher NO and Frame MC: Calpain: a role in cell transformation and migration. Int J Biochem Cell Biol 34: 1539-1543, 2002.

5. Perrin BJ and Huttenlocher A: Calpain. Int J Biochem Cell Biol 34: 722-725, 2002.
6. Dear N, Matena K, Vingron M, et al: A new subfamily of vertebrate calpains lacking a calmodulin-like domain: implications for calpain regulation and evolution. Genomics 45: 175-184, 1997.

7. Matena K, Boehm T and Dear N: Genomic organization of mouse Capn5 and Capn6 genes confirms that they are a distinct calpain subfamily. Genomics 48: 117-120, 1998.

8. Tonami $\mathrm{K}$, Kurihara Y, Aburatani $\mathrm{H}$, et al: Calpain 6 is involved in microtubule stabilization and cytoskeletal organization. Mol Cell Biol 2007 Jan 8 [Epub ahead of print].

9. Skubitz KM and Skubitz AP: Differential gene expression in uterine leiomyoma. J Lab Clin Med 141: 297-308, 2003.

10. Skubitz KM and Skubitz AP: Differential gene expression in leiomyosarcoma. Cancer 98: 1029-1038,2003.

11. Lee SJ, Choi YL, Lee EJ, et al: Increased expression of calpain 6 in uterine sarcomas and carcinosarcomas: an immunohistochemical analysis. Int J Gynecol Cancer 17: 248-253, 2007.

12. Campion MJ, McCance DJ, Cuzick J, et al: Progressive potential of mild cervical atypia: prospective cytological, colposcopic and virological study. Lancet 2: 237-240, 1986.

13. Ostor AG: Natural history of cervical intraepithelial neoplasia: a critical review. Int J Gynecol Pathol 12: 186-192, 1993.

14. Cox JT: Management of precursor lesions of cervical carcinoma: history, host defense and a survey of modalities. Obstet Gynecol Clin North Am 29: 751-785, 2002.

15. Klaes R, Friedrich T, Spitkovsky D, et al: Overexpression of p16(INK4A) as a specific marker for dysplastic and neoplastic epithelial cells of the cervix uteri. Int J Cancer 92: 276-284, 2001.

16. Lin WM, Michalopulos EA, Dhurander N, et al: Allelic loss and microsatellite alterations of chromosome 3 p14.2 are more frequent in recurrent cervical dysplasias. Clin Cancer Res 6: 1410-1414, 2000

17. Connolly DC, Greenspan DL, Wu R, et al: Loss of fhit expression in invasive cervical carcinomas and intraepithelial lesions associated with invasive disease. Clin Cancer Res 6: 3505-3510, 2000.

18. Bibbo M, Dytch HE, Alenghat E, et al: DNA ploidy profiles as prognostic indicators in CIN lesions. Am J Clin Pathol 92: 261-265, 1989.

19. Wisman GB, De Jong S, Meersma GJ, et al: Telomerase in (pre)neoplastic cervical disease. Hum Pathol 31: 1304-1312, 2000.

20. Goll DE, Thompson VF, Li H, et al: The calpain system. Physiol Rev 83: 731-801, 2003. 